NOTICE:

This is a paper that was accepted for publication in the IEEE Transactions on Human-Machine Systems. It is not the final published version. The DOI of the definitive version available in IEEE Xplore is http://dx.doi.org/10.1109/THMS.2017.2693684

(C) 2017 IEEE. Personal use of this material is permitted. Permission from IEEE must be obtained for all other uses, in any current or future media, including reprinting/republishing this material for advertising or promotional purposes, creating new collective works, for resale or redistribution to servers or lists, or reuse of any copyrighted component of this work in other works. 


\title{
Mobile Three-Dimensional Maps for Wayfinding in Large and Complex Buildings: Empirical Comparison of First-Person vs. Third-Person Perspective
}

\author{
Stefano Burigat, Luca Chittaro, and Riccardo Sioni
}

\begin{abstract}
The computational capabilities of today's smartphones make it possible to take advantage of mobile 3D maps to support navigation in the physical world. In particular, 3D maps might be useful to facilitate indoor wayfinding in large and complex buildings, where the typical orientation cues (e.g, street names) and location tracking technologies that can be used outdoors are unavailable. The use of mobile 3D maps for indoor wayfinding is still largely unexplored and research on how to best design such tools has been scarce to date. One overlooked but important design decision for 3D maps concerns the perspective from which the map content should be displayed, with first-person and third-person perspectives being the two major options. This paper presents a user study involving wayfinding tasks in a large and complex building, comparing a mobile 3D map with first-person perspective, a mobile 3D map with thirdperson perspective, and a traditional mobile 2D map. The firstperson perspective shows the mobile 3D map of the building from a floor-level egocentric point of view, while the third-person perspective shows the surroundings of the user from a fixed distance behind and above her position. Results of the study reveal that the mobile 3D map with third-person perspective leads to shorter orientation time before walking, better clarity ratings, lower workload, mental demand and effort scores, and higher preference score compared to the mobile 3D map with first-person perspective. Moreover, it leads to shorter orientation time before walking, better pleasantness ratings, lower mental demand scores, and higher preference score compared to the mobile 2D map.
\end{abstract}

Index Terms-Mobile, 3D maps, navigation, wayfinding, indoor, perspective, user study.

\section{INTRODUCTION}

$\mathbf{F}$ OR a growing number of users, mobile apps such as Google Maps and Apple Maps are an essential tool in supporting navigation of the physical world. Typically, these apps employ 2D maps to convey spatial knowledge of the environment [1], [2]. However, comprehending the spatial information provided by $2 \mathrm{D}$ maps and then transferring it to the egocentric experience of navigating the physical world may require a significant cognitive effort [3]-[5].

Compared with 2D maps, mobile 3D maps might theoretically simplify recognition of the objects contained in the environment and make it easier for the user to match what she sees in the map with what she sees in the physical world [6], [7]. This has motivated several researchers in the last few

S. Burigat, L. Chittaro, and R. Sioni are with the HCI Lab, Department of Mathematics, Computer Science and Physics, University of Udine, Italy years to explore the use of mobile 3D maps for navigation purposes [7]-[14]. However, the relatively few studies that have performed comparative evaluations of mobile $3 \mathrm{D}$ and $2 \mathrm{D}$ maps have found mixed results [9], [13], [15].

In general, prior research on the comparison of $2 \mathrm{D}$ and $3 \mathrm{D}$ visualizations in several fields (from information visualization [16] to aviation [17], from virtual reality [18] to cognitive science [19]) has revealed that the benefits of 3D visualizations depend upon the particular visualization design, the nature of the task, and the type of information to be displayed. This is why a proper answer to the question whether mobile 3D maps might provide advantages over their $2 \mathrm{D}$ counterparts can only be based on a systematic exploration of their design space and context of use.

The study we present in this paper is a step in the exploration of the design space for mobile 3D maps. More specifically, we focus on one of the most important but overlooked design choices for 3D maps, i.e., the perspective from which to show the map content. Perspective has been found to affect user performance in fields such as game studies [20][22] and teleoperation [23]-[25] but no empirical research on how different perspectives compare in terms of usability and effectiveness appears in the literature on mobile 3D maps for navigation. Drawing from existing 3D applications and games, we investigate the two most commonly used perspectives: (i) the first-person perspective that shows the 3D map from the same egocentric point of view the user has when she looks at the physical world and (ii) the third-person perspective that shows the 3D map from a fixed-distance viewpoint behind and above the user position. Most of the existing studies on mobile 3D maps concern either the first-person perspective [7], [9] or the third-person perspective [14], [15], [18], [26] but not both.

The second important choice we made in the study was to focus on indoor wayfinding in large and complex buildings. Our interest for this context of use was motivated by the following considerations:

- wayfinding, defined as the process of following a route or path from an origin to a destination through purposeful and goal directed travel [27], is one of the most common navigation tasks but has drawn surprisingly little attention in the literature on 3D maps,

- indoor navigation is drawing increasing commercial attention, as demonstrated by Google's projects on indoor maps [28] and mobile 3D-sensing technology [29], but 
most of the research effort to date has been aimed at exploring indoor location tracking technologies [30][32],

- the typical orientation cues that people rely on while navigating outdoors with a 2D map (i.e., street names and crossings) [13] are unavailable indoors,

- location tracking technology is not commonly available in most buildings; mobile navigation apps thus have to pass on the burden of matching map and physical world almost entirely to the user, a task that seems to be facilitated by 3D maps [7].

Overall, the study in this paper compares three mobile maps: a 3D map with a first-person perspective, a 3D map with a third-person perspective, and a traditional 2D map. To investigate the three maps, we developed a mobile app that displays the path the user has to follow to reach specific destinations inside buildings. We used the mobile app to support users wayfinding in a large and complex university building, collecting data about wayfinding performance as well as subjective user experience.

The paper is organized as follows. Section 2 reviews relevant literature on $2 \mathrm{D}$ and $3 \mathrm{D}$ visualizations and the effect of perspective in 3D maps. In Section 3, we describe the three maps we considered in the study and the app we used to display the maps and support wayfinding. Sections 4 and 5 illustrate in detail the user study and its results, while Sections 6 and 7 discuss the results and present conclusions and future work.

\section{RELATED WORK}

In this section, we first survey research on the effectiveness of $2 \mathrm{D}$ and $3 \mathrm{D}$ visualizations, with a focus on 2D and 3D maps as navigation support tools. Then, we review contributions on the effects of perspective on data visualization.

\section{A. Studies on $2 D$ vs. $3 D$ visualizations}

1) Mobile $2 D$ and $3 D$ maps for navigation in the physical world: A few studies in the literature have compared mobile 2D and 3D maps for outdoor navigation. The exploratory study presented in [7] found that a mobile 3D map allows for an easier and faster recognition of buildings with respect to a $2 \mathrm{D}$ map. A subsequent study [13] suggested that the results in [7] might have been affected by the lack of street names in the 2D map. Some works provide evidence that mobile 2D maps allow for faster initial user orientation and route finding [9], [26] and enable users to extract more information in less time compared to 3D maps [13]. Other studies found that mobile 3D maps have a higher positive impact on user satisfaction compared to 2D maps [9], [14].

Investigations of $2 \mathrm{D}$ vs. 3D maps for indoor navigation have been rare. In [33], participants preferred a 3D route visualization over a 2D map or graphical directional arrows to reach destinations in a building. The study presented in [15] revealed a higher user satisfaction with a 3D map compared to a $2 \mathrm{D}$ map for wayfinding in a multi-floor building.
2) $2 D$ and $3 D$ maps for navigation in virtual environments: Some studies on navigation in virtual environments (VEs) with 2D and 3D maps might be relevant to our work because of their focus on indoor scenarios and the ability of VEs to help people acquire spatial knowledge about real-world places [34]-[37].

The study presented in [38] revealed that a 2D map outperformed a 3D map in a search task for targets in a multifloor virtual building while no significant differences between the two conditions were found in a direction estimation task. In a related study [18] involving navigation of a multi-floor virtual building with a 2D map, a 3D map, or no map, results revealed that both maps improved direction estimation and vertical navigation accuracy compared to the no map condition and that users were more accurate in a map drawing task with the $2 \mathrm{D}$ map.

3) $2 D$ and $3 D$ maps in aviation: Navigation with $2 \mathrm{D}$ and 3D maps has been investigated to a certain extent in the aviation literature [6], [17], [39], [40]. These works were primarily aimed at studying the effect of different types of map presentation on operational tasks (e.g., traffic management, route evaluation, identification of objects, distance evaluation). Results revealed the superiority of 3D maps for qualitative judgments (e.g., identification of objects) and the superiority of 2D maps for quantitative judgments (e.g., relative positions and distance evaluations).

4) Scientific and information visualization: Several studies in the scientific and information visualization literature have compared 2D and 3D visualizations, with conflicting results. $3 \mathrm{D}$ visualizations have been found to outperform $2 \mathrm{D}$ visualizations in tasks involving graph comprehension [41] and web page retrieval [42]. Other studies showed no reliable performance difference between 2D and 3D visualizations of web content [43], [44]. A positive effect of 3D on spatial memory was found in an experiment about hierarchical 2D and 3D displays [45]. A later replication of the experiment did not find such effect [46]. In some specific domains, evidence has also been found that a $3 \mathrm{D}$ visualization can negatively affect user performance [47], [48].

5) Implications of $2 D$ vs. $3 D$ visualization studies: In general, studies on 2D vs. 3D visualizations found considerable variation in terms of task performance but found evidence of a significant user preference for $3 \mathrm{D}$ visualizations across different fields.

The results of scientific and information visualization studies might not be directly transferred to real world navigation because they involve tasks that rely on small-scale spatial abilities rather than the large-scale abilities required for navigation [49]. Results of aviation and VE studies support the hypothesis that 2D maps are more effective for decision making when dealing with relative position tasks while 3D maps are best used when dealing with shape perception decisions. The outdoor navigation studies hint at the possibility that 2D maps might suffer in comparison with 3D maps when position tracking technology and orientation cues such as street names are unavailable, a common scenario for indoor navigation. However, the few indoor navigation studies in the literature provide only limited insights on the effectiveness of mobile 3D 
maps, focusing on preference data and non real-time mobile 3D maps.

Our study will help to identify conditions under which mobile 3D maps might prove useful. In particular, we investigate whether real-time mobile 3D maps might be beneficial in a typical indoor wayfinding scenario, where users need to match what they see in the map with what they see in the physical world.

\section{B. Studies on the effect of perspective in $3 D$ environments}

Map perspective is an important design choice for 3D maps but has received no attention in the navigation literature. The navigation studies we surveyed considered either a first-person perspective [7], [9], or a third-person perspective [14], [15], [18], [26]. The only study that involved different perspectives [13] did not specifically focus on the effect of such feature.

1) Game studies: Perspective has been investigated to a certain extent in video game studies. In [50], the author argued that a first-person perspective in a $3 \mathrm{D}$ game improves player immersion, while the author of [51] stated that the thirdperson perspective provides the player with an experience of space that is closer to the real world. In a series of studies on driving tasks in video games, the authors of [52] found no performance difference between first- and third-person perspectives. The authors of [53] provide evidence that both first-person and third-person perspective support a high sense of embodiment during full-body reaching tasks. Other studies found that a first-person perspective causes higher immersion and more cognitive involvement [20], [22] while a third-person perspective yields more perceived control over the events in the 3D world [21].

2) Cognitive science: The literature on spatial knowledge acquisition includes the effect of perspective on the properties of the mental representations of an environment [37]. The authors of [54] found that learning a path in a 3D VE from a first-person perspective led to better navigation performance compared to a top-down perspective. The results presented in [55] revealed that a first-person perspective promotes efficient navigation of a 3D VE when people move through local and constrained space, while a top-down perspective improves performance when people navigate between distant places or must change route plans. In [19], the authors found evidence that the third-person perspective induces activation of areas of the brain associated to first- and top-down perspectives.

3) Augmented reality: Relevant studies on the effect of perspective on navigation come from augmented reality (AR) applications [56]. In [57], users preferred a combination of 2D map and AR over a 2D map only or AR only condition. However, there was no overall difference in task completion time among the three conditions. In [58], the authors found that a 3D map was perceived as a more reliable visualization for location and orientation estimation compared to an AR approach. In a series of experiments in which feeds of the real world were streamed to a Head-Mounted Display [59][61] the third-person perspective was preferred for real-world navigation and interaction with moving objects, while the firstperson perspective was preferred for fine manipulations of objects.
4) Teleoperation: In the teleoperation domain, the typical operator view on a remote vehicle is captured by the front camera mounted on the vehicle (first-person perspective). In [62], the authors report that operator performance increased when operators could use a top-down view of the environment. Several other studies [23]-[25] found that operators perform better in navigation tasks when using a third-person view of the vehicle rather than the front camera view.

5) Implications of perspective studies: The literature on the effect of perspective shows significant differences between first-person and third-person perspectives. While most game studies focused only on immersion and engagement rather than real-world navigation tasks, the results found in the augmented reality and teleoperation domains show that the third-person perspective is usually preferred and gives performance advantages in navigation tasks. Cognitive science studies provide a possible explanation of these advantages, showing that the third-person perspective might allow humans to be more efficient in storing and recalling spatial information.

Our study will contribute to clarify whether these results transfer to the mobile navigation context.

\section{THE CONSIDERED INDOOR WAYFINDING APP AND MAPS}

To support our study, we first developed an indoor wayfinding app for Android devices using the Unity game engine. The app displays 3D or 2D maps of the considered environment and the path to reach specific destinations. We then created three maps of the multi-floor university building where we carried out the study: a 3D map with first-person perspective, a 3D map with third-person perspective and a 2D map.

\section{A. The mobile indoor wayfinding app}

The developed app allows users to choose start and destination points from a list of predefined options and helps users reach the selected destination by highlighting on a map the path one has to follow. A path is composed of a sequence of segments connected by nodes, where each segment is displayed on the map as a series of yellow chevrons pointing in the direction users have to follow and each node is displayed as a yellow circle (Fig. 1a). A blue arrow, initially positioned on the first node of the path, marks the current location of the user. Internally, the app represents the sets of all nodes as a graph and can compute the shortest path between any two nodes using a version of the Dijkstra algorithm for the single-source shortest path problem.

The app does not rely on location tracking technology. The user moves from node to node along the path in the map by pressing the "Next node" button (bottom right in Fig. 1a). The transition to the next node is displayed as an animation showing the blue arrow moving, at a fixed speed, along the path segment connecting the current node with the next node. When the blue arrow reaches the next node, the path segment that connects to the previous node disappears, and the segment that has to be followed next is highlighted in orange (Fig. 1a). The "Previous node" button (bottom left in Fig. 1a) allows users to go back to the previous node in case of a mistake. 

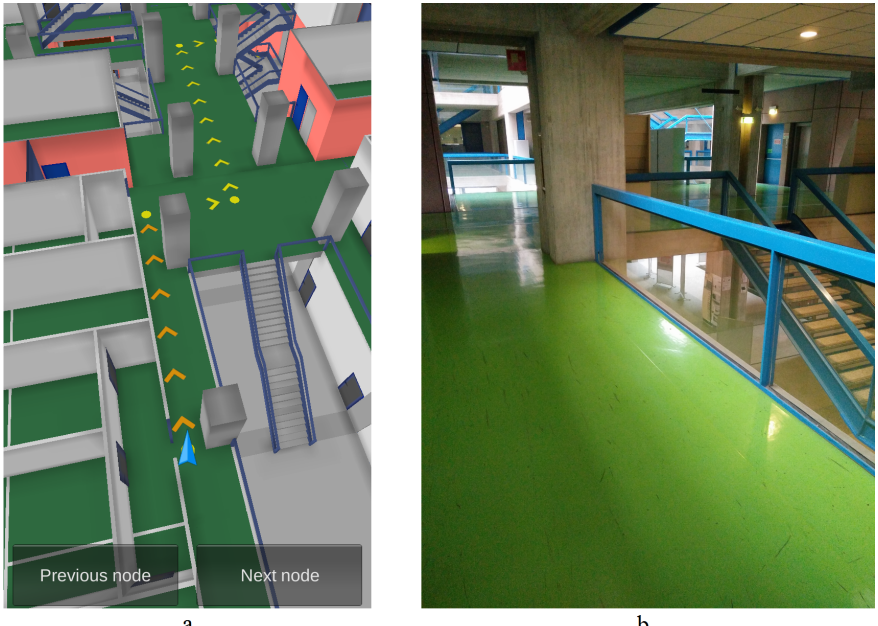

Fig. 1. (a) A screenshot of the indoor wayfinding app showing part of a path and the buttons for navigation. (b) A photo of the corresponding area in the real building.

Rotation of the map is automated, and depends on the physical orientation of the device obtained through the built-in compass of the smartphone.

The typical use of the app involves the following steps: (i) the user chooses start and destination points from a list of predefined options, (ii) the app computes the shortest path from start to destination point, showing it as a sequence of segments connected by nodes (Fig. 1a), (iii) the app assumes the user is positioned at the start point and waits for the user to press the "Next node" button, (iv) the user aligns the map in the direction of the next segment by rotating the device in that direction, (v) the user presses the "Next node" button, (vi) the app shows the animated transition to the next node; the transition shows the blue arrow that marks the user's position moving from the current node to the next node, (vii) during the animated transition, the user walks in the building to the position marked by the next node, (viii) the app waits for the user to tap on the "Next node" button. An iteration of steps (iv), (v), (vi), (vii), (viii) allows users to reach the destination.

\section{B. The considered maps}

As a first step in the creation of the maps, we imported 2D digital blueprints of the floors of the considered building in a desktop 3D modeling software (3ds Max). The blueprints were used as a reference to manually create a $3 \mathrm{D}$ model of the building with 3ds Max tools. We also walked the physical building to obtain information for the 3D model that was not available in the blueprints, such as materials and colors of walls, columns, doors and other useful internal elements of the building. Figure 2 shows an overview of the final model. The building is a large $(200 \mathrm{~m} \times 150 \mathrm{~m})$ and complex structure consisting of a ground floor with administration offices, classrooms and laboratories, a second and a third floor with offices and laboratories, and one underground floor with laboratories and classrooms. The building has a symmetric shape and a limited number of landmarks (most of which are located on the ground floor), making the physical navigation experience disorienting even after multiple visits.

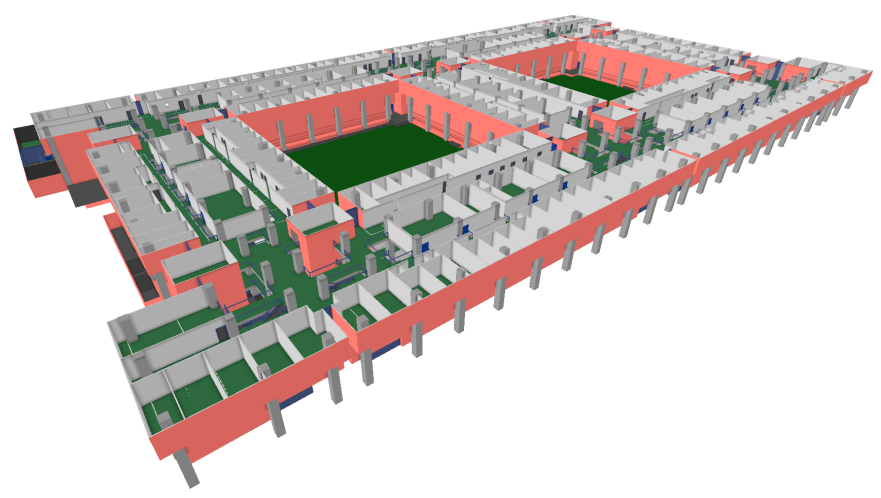

Fig. 2. The 3D model of the building selected for the study.

To improve performance of the mobile indoor wayfinding app, we focused on keeping the complexity of the 3D model as low as possible while preserving those visual details that might help users in understanding their position inside the building (concrete columns, color patterns on floors and walls, doors, staircases, elevators, etc.). To keep complexity low, we modeled each architectural element of the building with the minimum possible number of polygons, we included simplified versions of complex shapes (for example, columns in the real building are more complex than the simple parallelepipeds we used in the model), we used simple color patterns instead of textures whenever possible. We also generated pre-baked lightmaps to add visual realism to the $3 \mathrm{D}$ model without requiring real-time shading which would be too computationally expensive on many mobile phones.

To generate the three maps for the study, we imported the 3D model of the building in the Unity engine and wrote a script in Unity to conditionally change the camera parameters according to our needs. The camera in a Unity application is the (virtual) device through which the viewer looks at the environment (in our case, the 3D model of the building). The camera can be controlled through parameters such as position in the environment, pitch angle, and field of view. The script we wrote allowed us to define three sets of parameters for the same 3D model, one set for each map. We then created a basic interface that allowed us to choose one of the three sets of parameters at the start of the app and thus display the corresponding map.

The camera parameters for the three maps were chosen as follows:

- The 3D map with first-person perspective was obtained by positioning the camera in the 3D model at $1.8 \mathrm{~m}$ height from the floor, and at a pitch angle of $-15^{\circ}$ (Fig. $3 \mathrm{a}$ ).

- The 3D map with third-person perspective was obtained by positioning the camera in the 3D model at $15 \mathrm{~m}$ height from the floor, $2.6 \mathrm{~m}$ behind user position, and at a pitch angle of $-60^{\circ}$ (Fig. 3b). Additionally, all floors above the currently explored one were not rendered to avoid obstructions.

- The 2D map was obtained by positioning the camera in the $3 \mathrm{D}$ model at $30 \mathrm{~m}$ height from the floor, $7.5 \mathrm{~m}$ ahead 

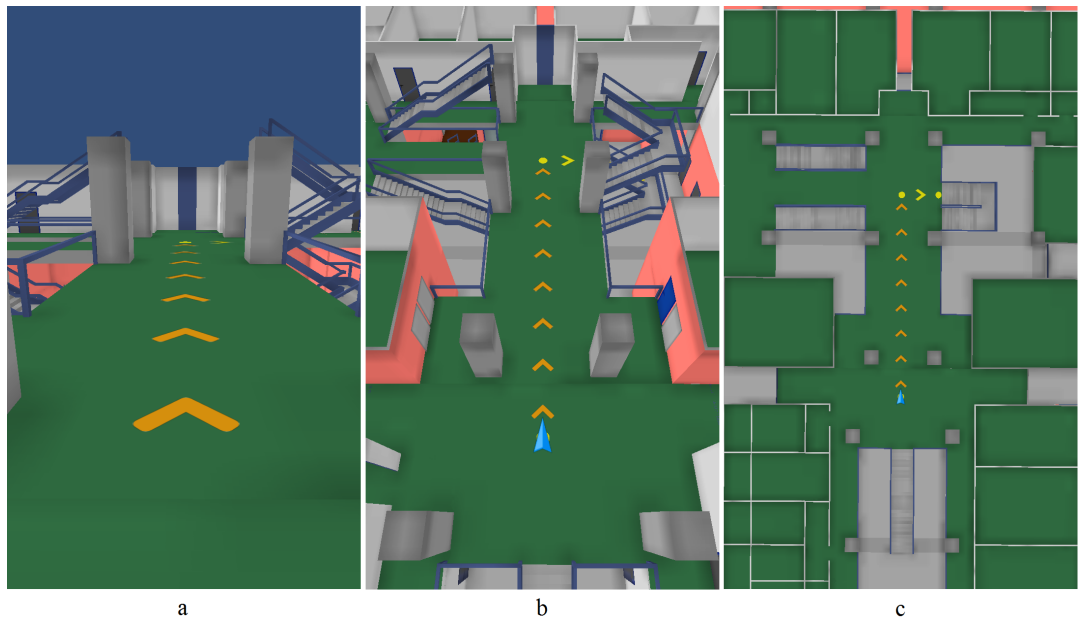

Fig. 3. The three maps at a given user location: (a) 3D map with first-person perspective; (b) 3D map with third-person perspective; (c) 2D map.

of the user position, and at a pitch angle of $-90^{\circ}$. We then used an orthographic projection to obtain the required 2D appearance (Fig. 3c). As in the 3D map with third-person perspective, floors above the currently explored one were not rendered to avoid obstructions.

For all three maps, the camera (vertical) field-of-view was set to $75^{\circ}$ to avoid distortions in the visualization of geometries and the camera $\mathrm{x}-\mathrm{y}$ coordinates were automatically set to center on the current segment of the path.

Due to the lack of specific guidelines in the literature, the values we chose for camera parameters derived from the type of perspectives we wanted to obtain and the following considerations on map design for indoor navigation and wayfinding. For the 3D map with first-person perspective, we recreated the point of view of an average-height adult (based on population statistics of our region) walking the floors of the building. This is representative of the typical first-person perspective used in current 3D applications and games. For the 3D map with third-person perspective, we aimed to let users see their surroundings and, at the same time, to make them clearly perceive important visual details of the map. Other camera parameters would have been possible, leading to third-person perspective variants such as the over-the-shoulder perspective of many action-adventure games or the birds-eye perspective of strategy games. For the 2D map, the goal was to show the area surrounding the user rather than provide an overview of the whole floor, which would be unreadable given the large size of the full building map. Similarly to the first- and thirdperson perspectives, the user position on the $2 \mathrm{D}$ map is not centered on the screen to present users with more information about the environment that is in front of them. As reported in [7], this decentralized position seems to reduce the cognitive workload of map reading. It is also worth noting that the 2D map retains important features of the 3D map such as colors and structures represented, unlike the simplified 2D maps used in other studies [15], [18].

An intrinsic consequence of the different camera parameters used is that each map shows a slightly different area of the building centered on the current segment of the path. The
3D map with first-person perspective shows only the area in front of the user, but possibly allows users to see farther in that direction compared to the other two maps. Due to its more elevated camera position, the 3D map with third-person perspective shows a slightly wider view than the 3D map with first-person perspective and provides more information about the area surrounding the user. Finally, the even higher camera position in the 2D map shows a wider area around the current segment of the path compared to the 3D map with third-person perspective.

\section{EXPERIMENTAL EVALUATION}

To compare the three maps, we carried out a user study in which participants had to navigate along three different paths in the considered building, each time using a different map, following the directions provided by the indoor wayfinding app. The study followed a within-subjects design with map (3D map with first-person perspective, 3D map with thirdperson perspective, 2D map) as independent variable. In the following, we will refer to the three conditions as 1P3D, 3P3D, and 2D, respectively.

\section{A. Participants}

The evaluation involved a sample of 78 participants $(67$ $\mathrm{M}, 11 \mathrm{~F})$ whose mean age was $22.00(\mathrm{SD}=1.86)$. They were recruited through direct contact among undergraduate Computer Science students at our university and received no compensation for their participation. On a 7-point scale $(1=$ no familiarity, 7 = very high familiarity), participants were familiar with 3D applications and games for mobile devices $(\mathrm{M}=5.46, \mathrm{SD}=1.40)$ and with mobile navigation applications $(\mathrm{M}=5.32, \mathrm{SD}=1.33)$. Participants mean spatial ability, assessed with the Santa Barbara Sense of Direction Scale (SBSOD) [63], was 4.40 ( $\mathrm{SD}=0.88)$. The SBSOD is a standardized questionnaire consisting of 15 statements about environmental spatial cognition. Participants indicated their level of agreement with each statement in the questionnaire on a 7-levels Likert scale ranging from 1 ("strongly agree") to 7 ("strongly disagree"). Positively stated items were reversed so that a 
higher average score across items indicates better spatial abilities.

\section{B. Materials}

The indoor wayfinding app was run on a Samsung Galaxy Nexus (Android 4.3) with 4.65", 720 x 1280 pixels screen. Logging code was used to record timing information about the events that could happen during app usage, such as the app starting or the user pressing the "Previous node" and "Next node" buttons. A stopwatch app running on a second smartphone was used by the experimenter to log the time participants spent walking or orienting themselves without walking, while they performed the task.

The three different paths participants followed in the building were of the same complexity in terms of length $(150 \mathrm{~m})$, number of segments (about 15), number of turns (about 15), number of transitions between floors (1) (see Fig. 4 for an example path). Each path required about three minutes to complete. The three paths were located in an area of the large building which was not familiar to Computer Science students because it contained Departments they had never visited before. We also selected the three paths in such a way that participants had only to walk for a short time to move from the end of a path to the starting point of the next path.

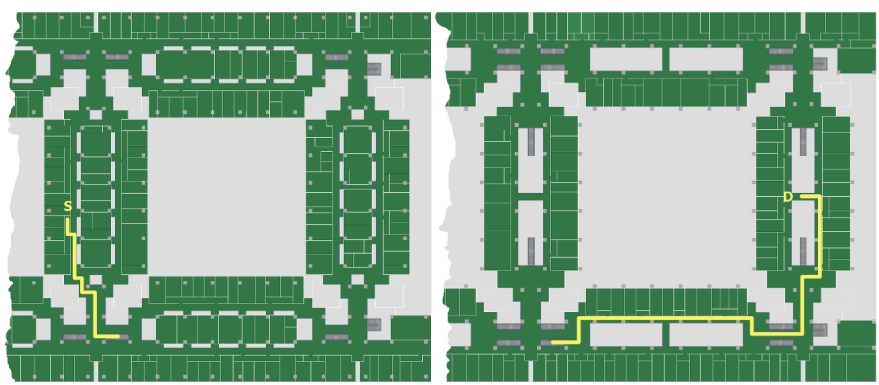

Fig. 4. Example of path (highlighted in yellow) followed by participants in the study. The path spans two floors, starting at point $S$ and ending at point D.

\section{Measures}

During the study, we collected data using questionnaires, logging code integrated in the wayfinding app, and the stopwatch application.

At the end of each path, we administered a questionnaire to obtain users' subjective assessment of the considered map in terms of clarity and pleasantness. Users indicated their level of agreement with each statement in the questionnaire on 7levels Likert items ranging from 1 ("strongly disagree") to 7 ("strongly agree"). To assess the general level of clarity of the information provided by each condition, participants rated the following items in the questionnaire: (i) "The directions are clearly presented by the app", (ii) "My current position is clearly presented on the map", (iii) "The path to follow is clearly presented", and (iv) "The on-screen map is clearly presented". The reliability of the scale was assessed with Cronbach's alpha $(0.84,0.79,0.80$ for $1 \mathrm{P} 3 \mathrm{D}, 3 \mathrm{P} 3 \mathrm{D}$, and $2 \mathrm{D}$, respectively). To assess participants' perception of the pleasantness of each condition, participants rated the following items: (i) "I found it pleasant to use the app", and (ii) "I found the app enjoyable". The reliability of the scale was assessed with Cronbach's alpha $(0.87,0.87,0.85$ for $1 \mathrm{P} 3 \mathrm{D}, 3 \mathrm{P} 3 \mathrm{D}$, and $2 \mathrm{D}$, respectively).

Then, we measured workload using the NASA Task Load Index (NASA-TLX) [64]. NASA-TLX derives an overall score based on the average of ratings on the following six subscales: mental demand, physical demand, temporal demand, performance, effort, and frustration level. Each sub-scale score can range in the 1-100 interval.

Through the stopwatch application and the logging code in the wayfinding application, we measured (i) wayfinding time, i.e., the total time it took participants to complete each path, and (ii) orientation time, i.e., the total time participants spent orienting themselves without walking along the path.

Finally, we administered a preference questionnaire that asked participants to rank the three conditions they had tested from the one they perceived as the best to the one they perceived as the worst, with ties allowed.

\section{Procedure}

Participants were first briefed about the experiment and asked to sign an informed consent if they agreed to participate. They were then asked to fill the initial demographic and SBSOD questionnaires. Participants were asked to walk at their normal walking speed during navigation and were not verbally informed about the paths so that they had to receive all information from the maps as they progressed. Participants were led to the start point of each path by the experimenter.

According to the within-subjects design, participants performed wayfinding tasks with all three map conditions (1P3D, 3P3D, and 2D). Before starting the wayfinding tasks, participants were asked to briefly familiarize with each type of map on three short paths that eventually took them near the starting point of the first experimental path. The order of the three maps during the familiarization session was the same that was employed during the experimental session. The order of three maps was counterbalanced and defined in such a way that all six possible combinations occurred every six participants. The order of the three paths was instead constant to minimize the transfer time between the end of a path and the beginning of the next path, and to avoid fixed associations between paths and maps. During the familiarization session, participants could ask the experimenter for clarifications about the app or the maps. The experimenter informed them that the smartphone compass might be inaccurate when walking near concrete columns or metallic objects (an intrinsic problem of magnetic compasses). Furthermore, the experimenter explained how the animation of the blue arrow along the path worked.

During the three experimental conditions, participants could not talk to the experimenter. The experimenter followed participants, keeping a few steps behind them until the destination was reached to avoid the possible risk of giving implicit guidance. The experimenter used the stopwatch app to log the time spent by participants walking and the time they spent orienting themselves without walking during the task. 
At the end of each wayfinding task, the experimenter asked participants to fill the questionnaire about the map, and the NASA-TLX. At the end of the experiment, participants were asked to fill the preference questionnaire. Finally, the experimenter collected participants' comments about the app and the maps with an informal interview.

\section{E. Hypotheses}

Due to the lack of studies on mobile 3D maps for indoor navigation, our investigation has an exploratory nature. Nevertheless, we had expectations for some of the outcomes based on the characteristics of indoor navigation, the features of the considered maps and the type of wayfinding task carried out by participants:

1) In terms of wayfinding and orientation time, we did not expect participants to perform worse with the 3D maps than with the $2 \mathrm{D}$ map. This was motivated by the lack in the 2D map of those orientation cues such as street names that seem to play a significant role in helping users read 2D maps [13]. Indeed, the only study involving mobile 3D maps where participants did not have access to street names for orientation [7] found better results for the 3D map condition compared to $2 \mathrm{D}$ maps.

2) We expected that the $3 P 3 D$ and the $2 \mathrm{D}$ conditions would score higher than the 1P3D condition in terms of clarity because they show a larger area of the building around the participants position and use an elevated camera that makes it easier to see the path to follow without obstructions.

3) We expected that both 3D maps would score higher than the 2D map in terms of pleasantness because of their higher visual realism and graphical richness compared to the 3D maps used in other studies on mobile indoor navigation [15], [18].

4) We hypothesized that participants would not perceive a higher workload wayfinding with the 3D maps compared to the 2D map, for the same reasons of hypothesis 1 .

5) Based on the results of [15] on user satisfaction with indoor 3D maps, we expected that the 3P3D condition would score higher that the $2 \mathrm{D}$ condition in terms of preference.

\section{RESULTS}

We used one-way repeated measures analysis of variance (ANOVA) to analyze wayfinding and orientation time, Friedman's test to analyze questionnaire ratings, workload scores, and preference, and Spearman's test to analyze correlations between variables. Before using ANOVA, we assessed data normality using the Shapiro-Wilk test. Significant effects were further explored with Tukey's multiple comparisons post-hoc test in the case of ANOVA and Dunn's multiple comparisons post-hoc test in the case of Friedman. When ANOVA reported a significant difference among conditions, we evaluated effect size with eta-squared $\left(\eta^{2}\right)$. When Friedman's test reported a significant difference among conditions, we evaluated effect

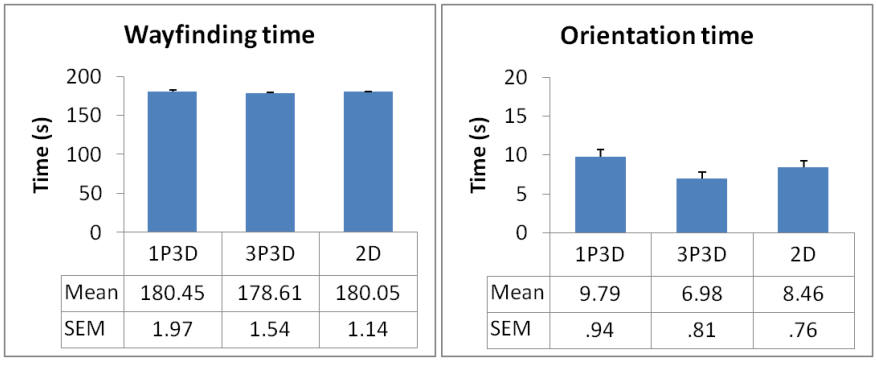

Fig. 5. Mean wayfinding and orientation time. Error bars indicate standard error of the mean (SEM).

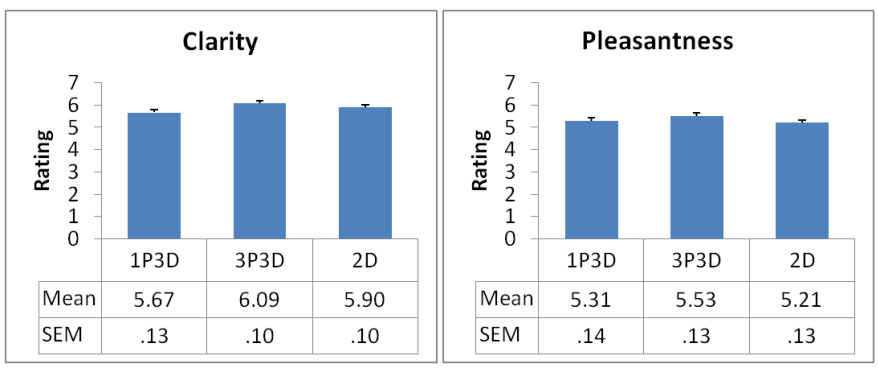

Fig. 6. Mean clarity and pleasantness score. Error bars indicate standard error of the mean (SEM).

size by computing the average Spearman's rho from Kendall's correlation coefficient W [65].

Figure 5 shows mean wayfinding and orientation time for the three map conditions. ANOVA on wayfinding time did not show a statistically significant difference among conditions $(F(2,154)=0.50, p>0.05)$. Since orientation time data were not normally distributed, we applied a log transformation [65] before using ANOVA with Greenhouse-Geisser correction, which revealed a statistically significant difference among conditions $\left(F(2.00,153.80)=7.61, p<0.001, \eta^{2}=0.09\right)$. The post-hoc Tukey's multiple comparisons test showed a significant difference $(p<0.001)$ between 1P3D and 3P3D, and a significant difference $(p<0.05)$ between 3P3D and 2D. The difference between 1P3D and 2D was small, and did not reach statistical significance.

Analysis of clarity and pleasantness ratings, whose means are shown in Fig. 6, showed significant differences among conditions in clarity $\left(\chi^{2}(2, N=78)=14.75, p<0.001\right.$, $\left.\bar{r}_{s}=0.08\right)$ as well as pleasantness $\left(\chi^{2}(2, N=78)=8.80\right.$, $\left.p<0.05, \bar{r}_{s}=0.04\right)$. The post-hoc Dunn's multiple comparisons test showed a significant difference in clarity $(p<0.01)$ between 1P3D and 3P3D. Differences between 1P3D and 2D, and between 3P3D and 2D did not reach statistical significance. Dunn's multiple comparison test showed also a significant difference in pleasantness $(p<0.05)$ between 3P3D and 2D. Differences between 1P3D and 3P3D, and between 1P3D and 2D did not reach statistical significance.

Figure 7 shows mean ratings of the NASA-TLX overall workload, mental demand and effort scales. The nonparametric Friedman's test on overall workload revealed a significant effect $\left(\chi^{2}(2, N=78)=10.23, p<0.01\right.$, $\left.\bar{r}_{s}=0.05\right)$ and Dunn's multiple comparisons post-hoc test showed a significant difference $(p<0.01)$ between 1P3D 


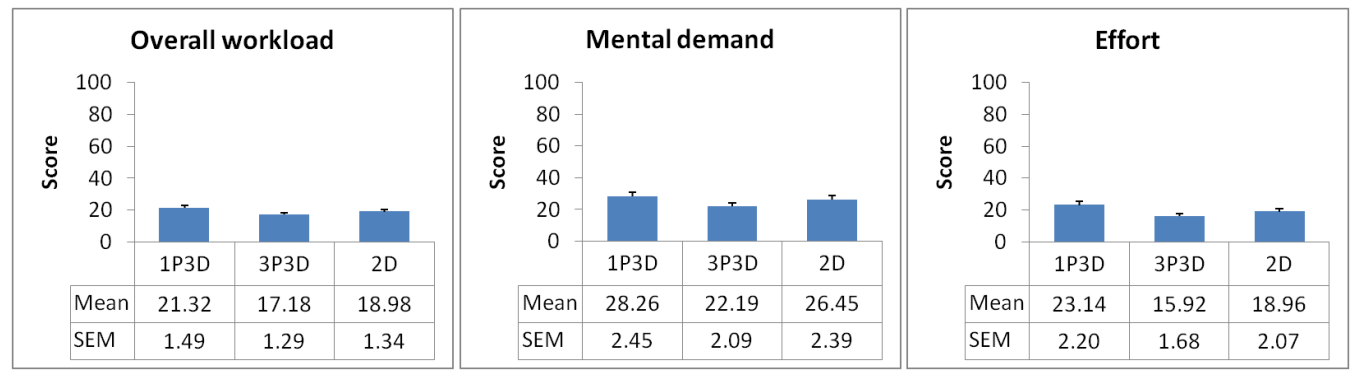

Fig. 7. Mean ratings for overall workload, mental demand, and effort. Error bars indicate standard error of the mean (SEM).

and 3P3D, with users experiencing less workload with 3P3D. Friedman's test on sub-scale ratings revealed a significant effect for mental demand $\left(\chi^{2}(2, N=78)=11.46, p<0.005\right.$, $\left.\bar{r}_{s}=0.06\right)$ and effort $\left(\chi^{2}(2, N=78)=9.12, p<0.05\right.$, $\left.\bar{r}_{s}=0.05\right)$. For mental demand, Dunn's post-hoc test showed a significant difference $(p<0.01)$ between 1P3D and 3P3D and a significant difference $(p<0.05)$ between 3P3D and 2D, with users experiencing less mental demand with 3P3D. For effort, Dunn's post-hoc test showed a significant difference $(p<0.05)$ between 1P3D and 3P3D, with users experiencing less effort with 3P3D. Differences among conditions for physical demand, temporal demand, performance, and frustration did not reach statistical significance.

Friedman's test on preference data, whose means are shown in Fig. 8, revealed a statistically significant difference among conditions $\left(\chi^{2}(2, N=78)=45.95, p<0.001, \bar{r}_{s}=0.29\right)$. Dunn's multiple comparisons tests showed a significant difference $(p<0.001)$ between 1P3D and 3P3D as well as a significant difference $(p<0.001)$ between 3P3D and 2D. The difference between 1P3D and 2D was small, and did not reach statistical significance.

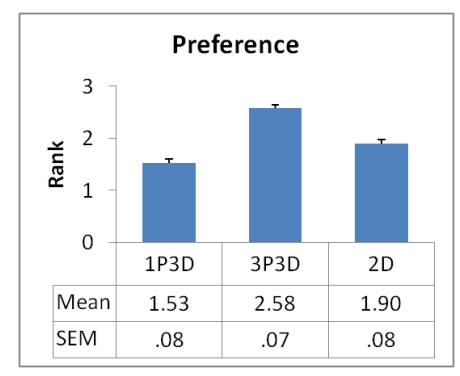

Fig. 8. Mean preference. Error bars indicate standard error of the mean (SEM).

Finally, Spearman's test found a strong negative correlation between workload and clarity $(\rho(78)=-0.46, p<0.001$ for 1P3D, $\rho(78)=-0.47, p<0.001$ for 3P3D, $\rho(78)=-0.58$, $p<0.001$ for 2D), between mental demand and clarity $(\rho(78)=-0.47, p<0.001$ for 1P3D, $\rho(78)=-0.45$, $p<0.001$ for 3P3D, $\rho(78)=-0.47, p<0.001$ for $2 \mathrm{D}$ ), and between effort and clarity $(\rho(78)=-0.34, p<0.001$ for 1P3D, $\rho(78)=-0.37, p<0.001$ for 3P3D, $\rho(78)=-0.38$, $p<0.001$ for $2 \mathrm{D}$ ) for all conditions. No statistically significant correlations were found between individual Santa Barbara score and performance data (wayfinding time and orientation time) for any of the conditions.

\section{DISCUSSION}

Unlike other studies in the literature [9], [13], [18], our study did not reveal any advantage of the 2D map compared to the two 3D maps. Instead, the 3P3D condition obtained the best results in several of the considered metrics. These results suggest that indoor 2D maps might suffer for the lack of orientation cues such as street names and crossroads that are crucial in users' outdoor navigation strategies [13].

Consistent with hypothesis 1, participants were able to reach their destinations in all three conditions without errors and taking the same total time. However, orientation time without walking was significantly lower in the 3P3D condition (about $29 \%$ lower compared to 1P3D, about $18 \%$ lower compared to 2D). A possible motivation for this result is that the thirdperson perspective makes it easier for participants to match the 3D map with the physical world, similarly to what was found in the aviation domain [6], [66], [67]. Interestingly, aviation studies focused on a different user group and a different navigation scenario compared to our study, which might hint at an effect that holds in general.

Qualitative observation and participants' comments revealed that most of the orientation time without walking was spent by participants trying to align their body in the direction of the next segment of the path after having reached a node. Three factors might have simplified the alignment process in the 3P3D condition compared to 1P3D. First, 3P3D allowed participants to see a larger area around their position. This might have provided orientation cues that participants in 1P3D could not obtain without orientation changes. The cost associated with the smaller area one can see from a first person perspective has been noticed in other studies [6]. Second, visibility of the path in 1P3D might have been lower because of the effects of perspective, with chevrons becoming progressively smaller with increasing distance from the participant's position. Third, the path to follow might not have been always fully visible in 1P3D due to obstructions by walls and columns. While this problem occurred in 3P3D as well, it might have affected 1P3D more seriously because of the previously mentioned lower path visibility. Compared to the 2D map, participants in the 3P3D condition did take advantage of additional orientation cues provided by the map. As suggested by the authors of [19], it is possible that the third-person perspective allows participants to use a mix of orientation strategies derived from both 2D maps and firstperson perspective. However, we do not have enough evidence 
to identify the specific cues participants did actually use in the 3P3D condition.

The lack of correlation between participants' spatial ability and navigation performance might be explained by the nature of the wayfinding task. Following a path probably requires only the visual ability to match map and physical world while more complex navigation tasks that involve the development and usage of a cognitive map [68] might benefit from higher spatial ability. Differences in the type of navigation task and required spatial knowledge might also explain the seeming inconsistencies between our results and those of other studies on 2D and 3D maps [13], [18], [38].

The higher clarity score for 3P3D compared to 1P3D (6.09 vs. 5.67) provides support for hypothesis 2 and was likely due to the explicit visualization of the participants' position and the larger unobstructed view of environment and path. However, it is harder to explain why no similar difference in clarity was found between 2D and $1 \mathrm{P} 3 \mathrm{D}$, considering that the 2D map provides the same benefits as 3P3D in terms of unobstructed view of the path and indication of the participants' position. It is possible that participants were influenced in their rating by the similarity between the 3D map and the physical world but it is hard to determine if there were other variables at play.

The difference in pleasantness between 3P3D and 2D (5.53 vs. 5.21) and the lack of difference between 1P3D and 2D partially support hypothesis 3 and seem to suggest that higher visual realism alone is not sufficient to make a difference in terms of pleasantness. While participants preferred the condition that allowed them to more easily navigate the building, it is also possible that they expected even more visual realism and graphical richness from a first-person perspective map because of its similarity to our natural point of view in the physical world.

Workload results were consistent with hypothesis 4 and confirmed that participants did not find the 3D maps more difficult to read than the 2D map. The third-person perspective did actually obtain better workload scores compared to the 2D map, probably because the orientation cues provided by the $3 \mathrm{D}$ map reduce the mental effort needed to match map and real world during wayfinding. The differences we found between 1P3D and 3P3D in overall workload (21.32 vs. 17.18), mental demand (28.26 vs. 22.19) and effort (23.14 vs 15.92) were likely explained by the higher difficulty participants had in understanding and exploiting the information in the 1P3D condition, as we previously discussed for orientation time without walking. This seems to be supported by the strong negative correlation we found between overall workload (and its mental demand and effort subratings) and clarity: the clearer the information provided by a condition, the lower the workload perceived by participants.

The higher preference for the 3P3D condition over the $2 \mathrm{D}$ condition (2.58 vs. 1.90) supports hypothesis 5 and is consistent with the findings in [15] where similar conditions were investigated. The preference for 3P3D over 1P3D (2.58 vs. 1.53) is similarly unsurprising and in agreement with the other results of the study.

\section{CONCLUSIONS}

In this paper, we investigated the use of mobile 3D maps to support indoor wayfinding, comparing two different mobile 3D maps and a traditional 2D map of a large and complex building in a path following task. Our study fills a gap in the literature, focusing on the unique challenges posed by indoor environments. In this context, we explored a research question that has not been examined before in the human navigation literature, namely whether the perspective used to view the content of a 3D map could have an effect on navigation. Results of our study highlight that mobile 3D maps can be at least as effective as the more frequently used 2D maps to support indoor wayfinding, and that a third-person perspective 3D map can outperform both a first-person perspective 3D map and a 2D map in terms of orientation time without walking, clarity, mental demand, and user preference.

Further investigation will be needed to more thoroughly understand the extent to which 3D maps can benefit users during indoor navigation. For example, the task we considered involved following wayfinding instructions displayed in the map in the form of a path. While this is a fairly typical and realistic task, it would be interesting to understand whether mobile 3D maps would be useful for different navigation goals such as learning the structure of an environment or searching for objects. Such goals have been considered before in other studies [13], [18], [38]. Additionally, there might be map features other than perspective that could play a significant role in supporting effective indoor navigation. For example, we minimized user interaction with the maps with the aim of making the wayfinding app immediately usable after a short familiarization phase and have better control of the experimental variables. However, adding the possibility to freely zoom the $2 \mathrm{D}$ map or change the camera parameters in the 3D maps would probably have an effect on the knowledge one could acquire from the maps. Finally, our study involved a specific sample of participants (young, mostly male, Computer Science students). Thus, our results cannot be easily generalized to other groups, such as older people [5], who differ in terms of spatial knowledge acquisition and smartphone use abilities.

Despite the need for additional studies to obtain a more comprehensive picture on the use of mobile 3D maps for indoor navigation, our results should suggest designers of mobile wayfinding apps to take into consideration the possibility to include in their apps third-person perspective mobile $3 \mathrm{D}$ maps in addition to or as a replacement of 2D maps. We have now deployed an updated version of the indoor wayfinding app in our university building (which has no positioning infrastructure). The updated version uses the thirdperson perspective 3D map, uses step identification to guide users along paths (automatically moving the position along each segment of the path based on step length), and provides a refined interface that gives textual directions to users in addition to the visualization of paths.

\section{REFERENCES}

[1] P. W. Thorndyke and B. Hayes-Roth, "Differences in spatial knowledge acquired from maps and navigation," Cognitive psychology, vol. 14, no. 4 , pp. 560-589, 1982. 
[2] T. Meilinger, C. Hölscher, S. J. Büchner, and M. Brösamle, "How much information do you need? schematic maps in wayfinding and self localisation," in Spatial Cognition V Reasoning, Action, Interaction. Springer, 2007, pp. 381-400.

[3] A. J. Aretz and C. D. Wickens, "The mental rotation of map displays," Human Performance, vol. 5, no. 4, pp. 303-328, 1992.

[4] P. Rau and Y. Wang, "A study of navigation support tools for mobile devices," Proc. HCI Int., Crete, Greece, June, pp. 22-27, 2003.

[5] J. Goodman, S. A. Brewster, and P. Gray, "How can we best use landmarks to support older people in navigation?" Behaviour \& Information Technology, vol. 24, no. 1, pp. 3-20, 2005.

[6] C. D. Wickens, "The when and how of using 2-d and 3-d displays for operational tasks," in Proceedings of the Human Factors and Ergonomics Society Annual Meeting, vol. 44, no. 21. SAGE Publications, 2000, pp. 3-403-3-406.

[7] A. Oulasvirta, A. Nurminen, and A.-M. Nivala, "Interacting with $3 \mathrm{~d}$ and 2d mobile maps: an exploratory study," Helsinki Institute for Information Technology April, vol. 11, 2007.

[8] I. Rakkolainen and T. Vainio, "A 3d city info for mobile users," Computers \& Graphics, vol. 25, no. 4, pp. 619-625, 2001.

[9] C. Kray, C. Elting, K. Laakso, and V. Coors, "Presenting route instructions on mobile devices," in Proceedings of the 8th international conference on Intelligent user interfaces. ACM, 2003, pp. 117-124.

[10] S. Burigat and L. Chittaro, "Location-aware visualization of vrml models in gps-based mobile guides," in Proceedings of the tenth international conference on 3D Web technology. ACM, 2005, pp. 57-64.

[11] V. Coors and A. Zipf, "Mona 3d-mobile navigation using 3d city models," LBS and Telecartography, 2007.

[12] A. Nurminen and A. Oulasvirta, "Designing interactions for navigation in 3d mobile maps," in Map-based mobile services. Springer, 2008, pp. $198-227$.

[13] A. Oulasvirta, S. Estlander, and A. Nurminen, "Embodied interaction with a $3 \mathrm{~d}$ versus $2 \mathrm{~d}$ mobile map," Personal and Ubiquitous Computing, vol. 13, no. 4, pp. 303-320, 2009.

[14] J. M. Noguera, M. J. Barranco, R. J. Segura, and L. Martínez, "A mobile 3d-gis hybrid recommender system for tourism," Information Sciences, vol. 215 , pp. 37-52, 2012.

[15] A. Lorenz, C. Thierbach, N. Baur, and T. H. Kolbe, "Map design aspects, route complexity, or social background? factors influencing user satisfaction with indoor navigation maps," Cartography and Geographic Information Science, vol. 40, no. 3, pp. 201-209, 2013.

[16] P. Irani and C. Ware, "Diagramming information structures using $3 \mathrm{~d}$ perceptual primitives," ACM Trans. Comput.-Hum. Interact., vol. 10, no. 1, pp. 1-19, Mar. 2003.

[17] M. St John, M. B. Cowen, H. S. Smallman, and H. M. Oonk, "The use of $2 \mathrm{~d}$ and $3 \mathrm{~d}$ displays for shape-understanding versus relativeposition tasks," Human Factors: The Journal of the Human Factors and Ergonomics Society, vol. 43, no. 1, pp. 79-98, 2001.

[18] H. Li and N. A. Giudice, "The effects of $2 \mathrm{~d}$ and $3 \mathrm{~d}$ maps on learning virtual multi-level indoor environments," in Proceedings of the 1st ACM SIGSPATIAL International Workshop on MapInteraction. ACM, 2013, pp. 7-12.

[19] J. Barra, L. Laou, J.-B. Poline, D. Lebihan, and A. Berthoz, "Does an oblique/slanted perspective during virtual navigation engage both egocentric and allocentric brain strategies?" PLOS ONE, vol. 7, no. 11, p. e49537, 2012.

[20] K. Kallinen, M. Salminen, N. Ravaja, R. Kedzior, and M. Sääksjärvi, "Presence and emotion in computer game players during 1st person vs. 3rd person playing view: Evidence from self-report, eye-tracking, and facial muscle activity data," Proceedings of the PRESENCE, pp. 187190, 2007.

[21] E. L. Schuurink and A. Toet, "Effects of third person perspective on affective appraisal and engagement: Findings from second life," Simulation \& Gaming, vol. 41, no. 5, pp. 724-742, 2010.

[22] A. Denisova and P. Cairns, "First person vs. third person perspective in digital games: Do player preferences affect immersion?" in Proceedings of the 33rd Annual ACM Conference on Human Factors in Computing Systems. ACM, 2015, pp. 145-148.

[23] M. Sugimoto, G. Kagotani, H. Nii, N. Shiroma, F. Matsuno, and M. Inami, "Time follower's vision: a teleoperation interface with past images," IEEE Computer Graphics and Applications, vol. 25, no. 1, pp. 54-63, 2005.

[24] J. T. Hing, K. W. Sevcik, and P. Y. Oh, "Development and evaluation of a chase view for uav operations in cluttered environments," vol. 57, no. $1-4$, pp. $485-503,2010$.
[25] D. Saakes, V. Choudhary, D. Sakamoto, M. Inami, and T. Lgarashi, A teleoperating interface for ground vehicles using autonomous flying cameras. IEEE Computer Society, 2013, pp. 13-19.

[26] K. Laakso, O. Gjesdal, and J. R. Sulebak, "Tourist information and navigation support by using $3 \mathrm{~d}$ maps displayed on mobile devices," in Proceedings of HCI in Mobile Guides Workshop, 2003, pp. 34-39.

[27] R. G. Golledge, Wayfinding behavior: Cognitive mapping and other spatial processes. JHU press, 1999.

[28] "Google indoor maps," www.google.com/maps/about/partners/ indoormaps/, accessed: 2016-06-07.

[29] "Project tango," get.google.com/tango/, accessed: 2016-06-07.

[30] H. Liu, H. Darabi, P. Banerjee, and J. Liu, "Survey of wireless indoor positioning techniques and systems," Systems, Man, and Cybernetics, Part C: Applications and Reviews, IEEE Transactions on, vol. 37, no. 6, pp. 1067-1080, 2007.

[31] Y. Gu, A. Lo, and I. Niemegeers, "A survey of indoor positioning systems for wireless personal networks," IEEE Communications Surveys \& Tutorials, vol. 11, no. 1, pp. 13-32, 2009.

[32] N. Fallah, I. Apostolopoulos, K. Bekris, and E. Folmer, "Indoor human navigation systems: A survey," Interacting with Computers, vol. 25 , no. 1, pp. 21-33, 2013

[33] F. Taher and K. Cheverst, "Exploring user preferences for indoor navigation support through a combination of mobile and fixed displays," in Proceedings of the 13th International Conference on Human Computer Interaction with Mobile Devices and Services. ACM, 2011, pp. 201210.

[34] B. G. Witmer, J. H. Bailey, B. W. Knerr, and K. C. Parsons, "Virtual spaces and real world places: transfer of route knowledge," International Journal of Human-Computer Studies, vol. 45, no. 4, pp. 413-428, 1996.

[35] E. Arthur, P. Hancock, and S. Chrysler, "The perception of spatial layout in real and virtual worlds," Ergonomics, vol. 40, no. 1, pp. 69-77, 1997.

[36] P. N. Wilson, N. Foreman, and M. Tlauka, "Transfer of spatial information from a virtual to a real environment," Human Factors: The Journal of the Human Factors and Ergonomics Society, vol. 39, no. 4, pp. 526531, 1997.

[37] A. E. Richardson, D. R. Montello, and M. Hegarty, "Spatial knowledge acquisition from maps and from navigation in real and virtual environments," Memory \& cognition, vol. 27, no. 4, pp. 741-750, 1999.

[38] L. Chittaro and S. Venkataraman, "Navigation aids for multi-floor virtual buildings: A comparative evaluation of two approaches," in Proceedings of the ACM symposium on Virtual Reality Software and Technology. ACM, 2006, pp. 227-235.

[39] C. D. Wickens, C.-C. Liang, T. Prevett, and O. Olmos, "Electronic maps for terminal area navigation: Effects of frame of reference and dimensionality," The international journal of aviation psychology, vol. 6 , no. 3, pp. 241-271, 1996.

[40] S. Dixon, E. Fitzhugh, and D. Aleva, "Human factors guidelines for applications of 3d perspectives: a literature review," in SPIE Defense, Security, and Sensing. International Society for Optics and Photonics, 2009 , pp. $73270 \mathrm{~K}-73270 \mathrm{~K}$.

[41] C. Ware and G. Franck, "Evaluating stereo and motion cues for visualizing information nets in three dimensions," ACM Transactions on Graphics (TOG), vol. 15, no. 2, pp. 121-140, 1996.

[42] G. Robertson, M. Czerwinski, K. Larson, D. C. Robbins, D. Thiel, and M. Van Dantzich, "Data mountain: using spatial memory for document management," in Proceedings of the 11th annual ACM symposium on User interface software and technology. ACM, 1998, pp. 153-162.

[43] K. Risden, M. P. Czerwinski, T. Munzner, and D. B. Cook, "An initial examination of ease of use for $2 \mathrm{~d}$ and $3 \mathrm{~d}$ information visualizations of web content," International Journal of Human-Computer Studies, vol. 53, no. 5, pp. 695-714, 2000.

[44] A. Cockburn and B. McKenzie, " $3 \mathrm{~d}$ or not $3 \mathrm{~d}$ ?: evaluating the effect of the third dimension in a document management system," in Proceedings of the SIGCHI conference on Human factors in computing systems. ACM, 2001, pp. 434-441.

[45] M. Tavanti and M. Lind, "2d vs 3d, implications on spatial memory," in Information Visualization, 2001. INFOVIS 2001. IEEE Symposium on. IEEE, 2001, pp. 139-145.

[46] A. Cockburn, "Revisiting 2d vs 3d implications on spatial memory," in Proceedings of the fifth conference on Australasian user interfaceVolume 28. Australian Computer Society, Inc., 2004, pp. 25-31.

[47] H. Smallman, M. S. John, H. Oonk, and M. Cowen, "Track recognition using two-dimensional symbols or three-dimensional realistic icons," DTIC Document, Tech. Rep., 2000.

[48] M. Hicks, C. O'Malley, S. Nichols, and B. Anderson, "Comparison of $2 \mathrm{~d}$ and $3 \mathrm{~d}$ representations for visualising telecommunication usage," Behaviour \& Information Technology, vol. 22, no. 3, pp. 185-201, 2003. 
[49] M. Hegarty, D. R. Montello, A. E. Richardson, T. Ishikawa, and K. Lovelace, "Spatial abilities at different scales: Individual differences in aptitude-test performance and spatial-layout learning," Intelligence, vol. 34, no. 2, pp. 151-176, 2006.

[50] R. Rouse III, "What's your perspective?" ACM SIGGRAPH Computer Graphics, vol. 33, no. 3, pp. 9-12, 1999.

[51] L. N. Taylor, "Video games: Perspective, point-of-view, and immersion," Ph.D. dissertation, University of Florida, 2002.

[52] S. Bateman, A. Doucette, R. Xiao, C. Gutwin, R. L. Mandryk, and A. Cockburn, "Effects of view, input device, and track width on video game driving," in Proceedings of Graphics Interface 2011. Canadian Human-Computer Communications Society, 2011, pp. 207-214.

[53] H. G. Debarba, E. Molla, B. Herbelin, and R. Boulic, "Characterizing embodied interaction in first and third person perspective viewpoints," in 3D User Interfaces (3DUI), 2015 IEEE Symposium on. IEEE, 2015, pp. 67-72.

[54] F. Pazzaglia and H. A. Taylor, "Perspective, instruction, and cognitive style in spatial representation of a virtual environment," Spatial Cognition and Computation, vol. 7, no. 4, pp. 349-364, 2007.

[55] T. T. Brunyé, A. Gardony, C. R. Mahoney, and H. A. Taylor, "Going to town: Visualized perspectives and navigation through virtual environments," Computers in Human Behavior, vol. 28, no. 1, pp. 257-266, 2012.

[56] J. Huang, C.-H. Tsai, and S.-T. Huang, "The next generation of gps navigation systems," Communications of the ACM, vol. 55, no. 3, pp. 84-93, 2012.

[57] A. Dünser, M. Billinghurst, J. Wen, V. Lehtinen, and A. Nurminen, "Exploring the use of handheld ar for outdoor navigation," Computers \& Graphics, vol. 36, no. 8, pp. 1084-1095, 2012.

[58] A. Möller, M. Kranz, R. Huitl, S. Diewald, and L. Roalter, "A mobile indoor navigation system interface adapted to vision-based localization," in Proceedings of the 11th International Conference on Mobile and Ubiquitous Multimedia. ACM, 2012, p. 4.

[59] P. Salamin, D. Thalmann, and F. Vexo, "The benefits of third-person perspective in virtual and augmented reality?" in Proceedings of the ACM symposium on Virtual reality software and technology. ACM, 2006, pp. 27-30.

[60] — "Improved third-person perspective: a solution reducing occlusion of the 3pp?" in Proceedings of The 7th ACM SIGGRAPH International Conference on Virtual-Reality Continuum and Its Applications in Industry. ACM, 2008, p. 30.

[61] P. Salamin, T. Tadi, O. Blanke, F. Vexo, and D. Thalmann, "Quantifying effects of exposure to the third and first-person perspectives in virtualreality-based training," Learning Technologies, IEEE Transactions on, vol. 3, no. 3, pp. 272-276, 2010.

[62] N. Shiroma, N. Sato, Y.-h. Chiu, and F. Matsuno, "Study on effective camera images for mobile robot teleoperation," in Robot and Human Interactive Communication, 2004. ROMAN 2004. 13th IEEE International Workshop on. IEEE, 2004, pp. 107-112.

[63] M. Hegarty, A. E. Richardson, D. R. Montello, K. Lovelace, and I. Subbiah, "Development of a self-report measure of environmental spatial ability," Intelligence, vol. 30, no. 5, pp. 425-447, 2002.

[64] S. G. Hart and L. E. Staveland, "Development of nasa-tlx (task load index): Results of empirical and theoretical research," Advances in psychology, vol. 52, pp. 139-183, 1988.

[65] B. H. Cohen, Explaining psychological statistics. John Wiley \& Sons, 2008.

[66] J. C. Hickox and C. D. Wickens, "Effects of elevation angle disparity, complexity, and feature type on relating out-of-cockpit field of view to an electronic cartographic map." Journal of Experimental Psychology: Applied, vol. 5, no. 3, p. 284, 1999.

[67] B. T. Schreiber, C. D. Wickens, G. J. Renner, J. D. Alton, and J. C. Hickox, "Navigational checking using 3d maps: The influence of elevation angle, azimuth, and foreshortening," Human Factors: The Journal of the Human Factors and Ergonomics Society, vol. 40, no. 2, pp. 209-223, 1998.

[68] E. C. Tolman, "Cognitive maps in rats and men." Psychological review, vol. 55 , no. 4 , p. $189,1948$.

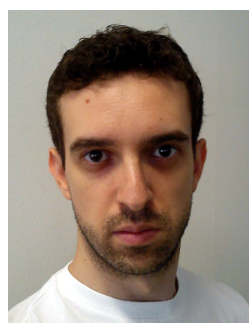

Stefano Burigat received the Ph.D. degree in Computer Science from the University of Udine, Italy, in 2007. He is currently Assistant Professor in the Department of Mathematics, Computer Science, and Physics of the University of Udine, Italy, where is member of the Human-Computer Interaction Laboratory (http://hcilab.uniud.it). His research interests include information presentation on mobile devices, mobile interface design and evaluation, positioning techniques for indoor and outdoor navigation, navigation aids for $3 \mathrm{D}$ virtual environments.

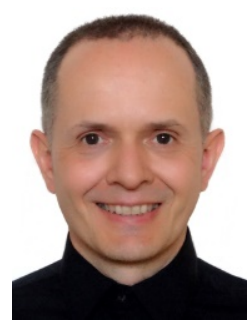

Luca Chittaro is Full Professor of Human Computer Interaction (HCI) in the Department of Mathematics, Computer Science, and Physics of the University of Udine, Italy, where he heads the $\mathrm{HCl}$ Lab (http://hcilab.uniud.it). He has authored or coauthored over 180 international academic publications, and he is an ACM Distinguished Speaker. His major research interests are in virtual reality, mobile $\mathrm{HCI}$, serious games, persuasive technology and their applications in health and safety. He has received research grants from a wide range of organizations, including the US Federal Aviation Administration (FAA), the European Union (EU), the Italian Ministry of University and Research (MIUR), and companies such as the Benetton Group and the Intesa Sanpaolo Bank group.

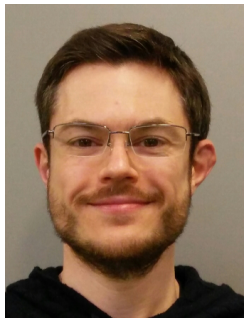

Riccardo Sioni received the Ph.D. degree in Computer Science from the University of Udine, Italy, in 2014. He is currently a Postdoctoral Researcher in the Department of Mathematics, Computer Science, and Physics of the University of Udine, Italy, where is member of the Human-Computer Interaction Laboratory (http://hcilab.uniud.it). His research interests include virtual reality, serious games, and psychophysiology. 